\title{
Nitrogen-Galinstan Two Phase Pumping for MHD Power Generation Systems
}

\author{
Josh Rosettani ${ }^{1}$, Philip Geddis ${ }^{2}$, Lijun $\mathrm{Wu}^{2}$, Bruce Clements ${ }^{2}$, Wael Ahmed ${ }^{1}$ \\ ${ }^{1}$ University of Guelph \\ 50 Stone Rd E, Guelph, Canada \\ jrosetta@uoguelph.ca; ahmedw@uoguelph.ca \\ ${ }^{2}$ CanmetENERGY-Ottawa, Natural Resources Canada \\ 1 Haanel Dr, Ottawa, Canada
}

\begin{abstract}
The continuous demand for energy has open many pathways to develop new sustainable power generation processes. Magnetohydrodynamics (MHD) power generation is an alternative process being investigated to recover energy from low temperature sources but is not limited to low temperatures. Liquid Metal MHD (LMMHD) power generation is the process of circulating liquid metal through an MHD generator using gas injection. The need to design a low energy two-phase gas-lift pump and evaluate its performance as well as the two-phase dynamics in this energy recovering process are crucial components to develop the system. In this study, a preliminary analysis utilizing ANSYS Fluent ${ }^{\circledR}$ for pumping two-phase flow mixture of nitrogen and galinstan is performed. These numerical results along with the analytical model based on drift flux assumptions are used to understand the performance of the gas-lift two-phase flow pump in this system. Reasonable agreement between the numerical and analytical results were obtained. The CFD results were used to describe the void fraction of the two phases in the pipe riser and considered to be the first step to understand the performance of LMMHD power generation loop.
\end{abstract}

Keywords: Two-Phase Flow; Liquid Metal; MHD system; CFD; Gas-lift pump.

\section{Introduction}

Power generation by MHD is the process of generating electricity from the basis of Faraday's law of electrodynamic induction. The basic principle of Faraday's law states that a moving conductor in a magnetic field will induce an electromotive force (EMF) and so current is produced [1]. In MHD power systems, the typical solid conductors are replaced by an electrically conducting fluid that flows through a magnetic field created by an MHD generator to induce an EMF [2]. Thus, the EMF created will generate a current in form of DC electricity which could be converted to AC. There are three main systems of MHD power generation: open cycle systems, closed cycle systems and closed cycle liquid metal systems. In this study, the liquid metal MHD (LMMHD) loop is under investigation. The LMMHD power system uses the principles of two-phase flow to circulate the liquid metal in a vertical closed loop. A gas is introduced to the liquid metal through a mixer device attached to one side of the vertical closed loop. Due to the differences in densities, the gas-liquid mixture on the mixer side will flow upwards in the vertical tube [3]. The gas-liquid mixture will separate at the top of the loop and liquid metal will travel back towards the mixer device through the down comer tube due to gravity and pass through the MHD generator $[4,5]$. The main function of the liquid metal in the loop is to serve as an electroconductive medium for which the current can pass through as it travels through the MHD generator. Due to the simplicity of the design as well as the versatility of liquid metal, MHD power systems have many advantages over conventional power generation systems. With no moving mechanical components in the closed cycle system there are none of the problems which commonly occur from bearing/lubrication issues in more conventional turbines and generators [6].

The main purpose of the mixer in the LMMHD loop is to introduce the gas into the riser where it interacts with the liquid metal to produce a lift within the riser tube. The formation of the bubbles created from the mixer have a crucial role in the overall efficiency of the system. Ideally, a higher quantity of larger size bubbles in the riser generates a better overall power conversion in the system [7]. In LMMHD flow systems, the most important parameter to be studied is the two-phase nature in the riser tube. To understand and properly predict the two-phase flow patterns occurring in the riser will help aid in improving the overall efficiency of the system [1]. Typically, LMMHD power systems found in literature use a perforated 
gas-injection nozzle as the mixer component. An improved two-phase mixing pump using dual injection methods has been developed by FloNergia Inc. for air-water applications. However, the application of the two-phase mixing pump has not yet been applied to liquid metal-gas flow mixtures. This study will utilize the design concept of FloNergia's FloMov Airlift Pump to numerically simulate galinstan-nitrogen in a vertical riser tube to better understand the two-phase flow characterises in an LMMHD loop. The performance results simulated through ANSYS fluent will be compared to an analytical model to provide an initial comparison of the predicted results between the two type of models.

\section{Analytical Model}

In order to access assess the performance of an airlift pump, an analytical model was developed by Kassab et al. [8] to used here to predict the performance of the gas-lift pump. The model assumes one dimensional flow in a vertical pipe and is dependent on the momentum, continuity, slip ratio, and friction factor equations that are solved iteratively. Stenning and Martin [9] combined the momentum equation and the continuity equation for a gas-lift case, which is presented as follows:

$$
\begin{gathered}
\frac{H_{s}}{L}-\frac{1}{\left(1+\frac{Q_{g}}{S Q_{L}}\right)}=\frac{V_{1}^{2}}{2 g L}\left[(K+1)+(K+2) \frac{Q_{g}}{Q_{L}}\right] \\
K=\frac{4 f L}{D}
\end{gathered}
$$

where $H_{s}$ is the static head, $L$ is the total pipe length, $Q_{g}$ and $Q_{l}$ are the volumetric flow rates of the gas and liquid phase respectively, $V_{1}$ is the superficial velocity at the pump riser inlet and $\mathrm{D}$ is the pipe diameter. The slip ratio, s, suggested by Griffith and Wallis [10] taking into consideration both liquid and gas volumetric flow rates as:

$$
s=1.2+0.2 \frac{Q_{g}}{Q_{L}}+\frac{0.35 \sqrt{g D}}{V_{1}}
$$

The friction factor in Eqn. (1) is calculated from Haaland [11] as:

$$
\frac{1}{\sqrt{f}}=-2.0 \log \left(\frac{\varepsilon / D}{3.7}+\frac{2.51}{R e \sqrt{f}}\right)
$$

where $\varepsilon$ is the pipe roughness and $R e$ is the Reynolds Number based on the mixture velocity. In the present study, an analytical model is developed for nitrogen-galinstan two phase flow in a vertical pipe.

\section{CFD Model}

In this study, an airlift pump designed by FloNergia is used. Three-dimensional numerical simulation was performed using ANSYS Fluent ${ }^{\circledR}$. A two-phase Volume of Fluid (VOF) model was applied for nitrogen and user-defined galinstan in an isothermal system. This type of model was chosen based on previous studies and the capabilities to more accurately predict the two-phase flow patterns occurring in a vertical riser pipe [12]. The standard K- $\varepsilon$ turbulence model was applied due to its ability to accurately represent a wide range of turbulent flows [13]. It is also important to model the surface tension forces acting between the surfaces of the two-phases to accurately predict the flow structure in the riser. A continuum surface model (CSF) was also used to capture the interaction between the two phases. Liu et al. [14] experimentally determined the surface tension between galinstan and nitrogen to be $0.534 \mathrm{~N} / \mathrm{m}$. 
A non-conformal mesh was applied to the fluid domain of the gas-lift pump and the pipe riser shown in figure 1. This meshing technique allowed for a more flexible but precise mesh to be applied to a complex geometry. Mesh refinement was applied to areas subject to more complex flow such as the wall boundaries and gas injection holes by using a bias factor to achieve the desired y+ parameter. The gas injection holes will first see the mixture of the two phases and so it is of high importance to accurately capture the flow structure. A y+ parameter of 15 was accepted in the present analysis given the complex geometry and turbulence model chosen for this domain.

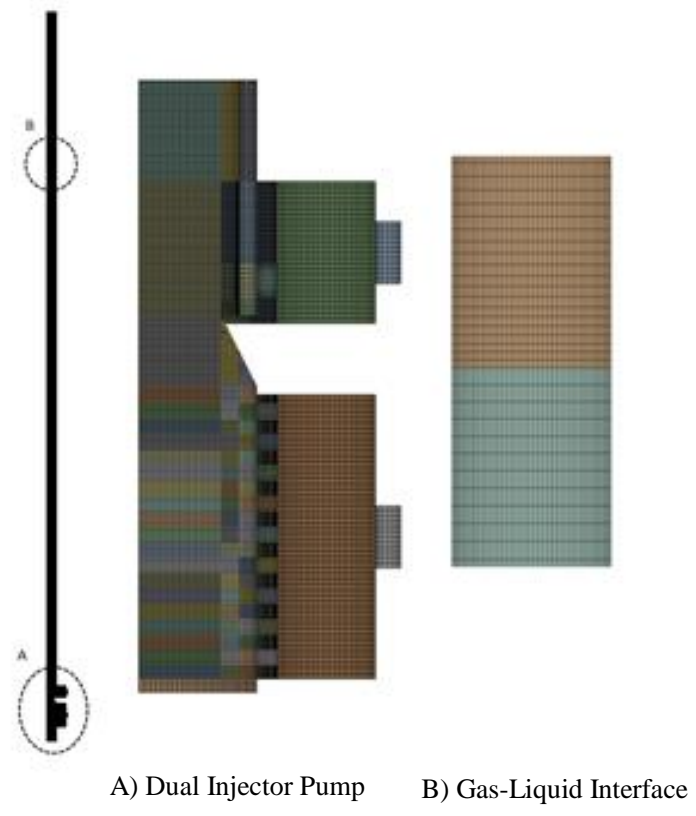

Fig. 1: Mesh for Dual Injector Gas-lift Pump Coupled with Vertical Riser.

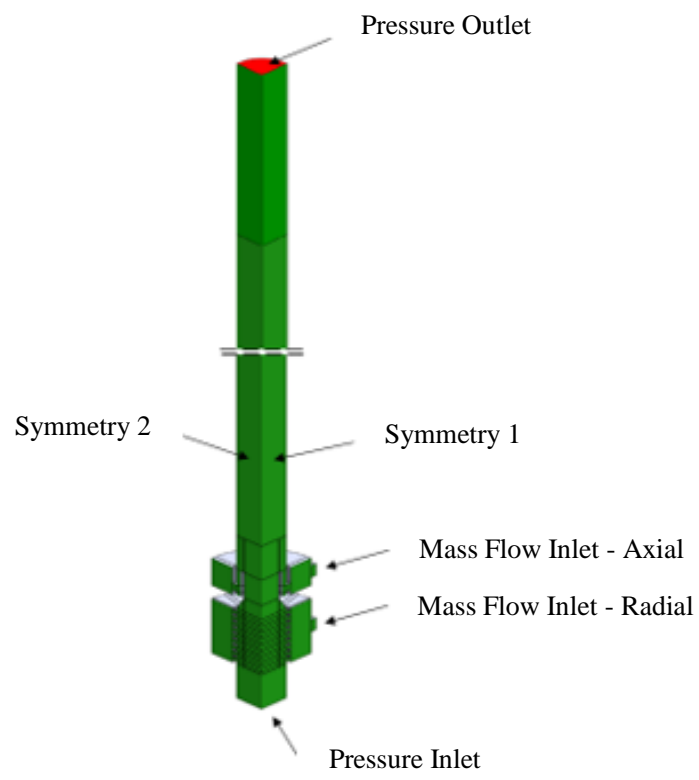

Fig. 2: Boundary Conditions of Gas-lift System.

In order to reduce computational time, a quarter of the 3-D airlift pump model was solved assuming symmetrical boundary conditions. Mass flow inlets at both injection points and pressure inlet boundary conditions were applied at the riser bottom. Also, a pressure outlet was applied at the top part of the riser shown in figure 2 .

\section{Results and Discussions}

A 1.6" inch gas-lift pump system was simulated for a range of nitrogen flow rates under a submergence ratio of $90 \%$. The submergence ratio is defined as the static head divided by the total length of the pipe. Simulations for a range of nitrogen mass flow rates between $0.0002-0.013 \mathrm{~kg} / \mathrm{s}$ were computed to obtain the full range of the gas-lifts performance. Each CFD simulation point was initialized using the results obtained from the steady state simulation. Once the initial values are properly initialized, the transient simulations of 5 seconds of time is used with a time step size of $1 \times 10^{-4}$ for a total of 50,000 is used. Each time step required 60 inner iterations to ensure an appropriate residual was achieved of 0.01 . 


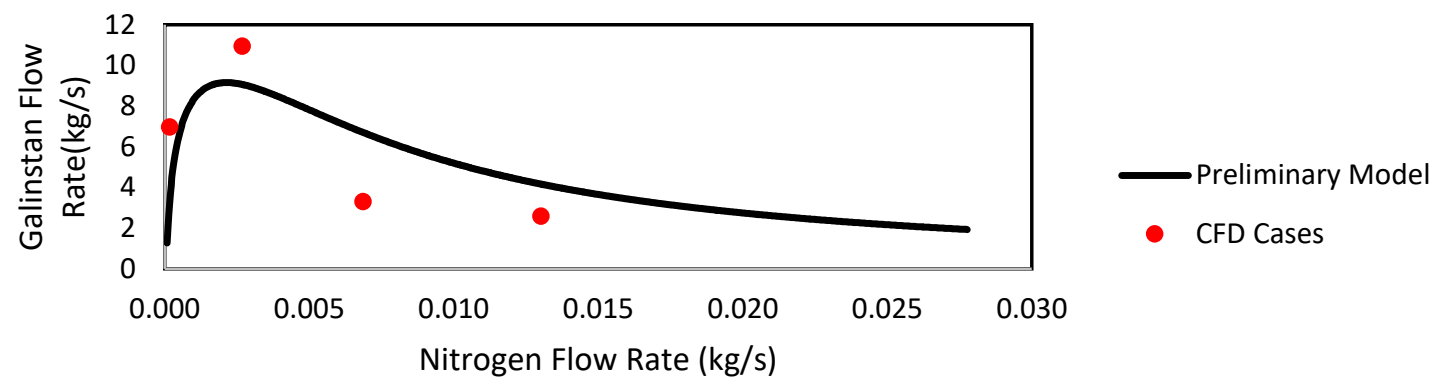

Fig. 3: Comparison of CFD results to Analytical Model.

The performance of a gas-lift system can be evaluated by comparing the input of gas into the pump to the output of liquid flow. Therefore, the liquid metal outflow was monitored by generating a report file for the time averaged liquid flow rate at the outlet of the riser. The results from the CFD simulations are compared to the analytical model and displayed in figure 3. It can be observed that as the gas injection increases, initially the liquid outflow increases rapidly until it hits a peak performance. After this peak the more gas injected will decrease the liquid outflow. This phenomenon often occurs because of the increase of frictional pressure drop due to the increasing gas-flow rate. This trend is seen in both the analytical model and CFD results, showing good agreement between the numerical model and analytical model. The percentage RMS error calculated between the values obtained from the analytical model and CFD results was found to be $\pm 15 \%$. To further describe the performance of the gas-lift pump, the void space contours at specific instances at time were plotted. It is effective to show the void space contours as it is tightly related to the performance of the gas-lift pump by understanding the two-phase flow pattern occurring in the riser. Figure 4 displays the void space contours for the CFD simulation of the first point on the performance graph at a nitrogen flow rate of $0.0002 \mathrm{~kg} / \mathrm{s}$. It is important for the formation of nitrogen gas bubbles to create larger gas bubbles that will act as a pneumatic piston to pump the liquid metal. Figure 4 shows that the flow upon exit of the pump starts to accumulate further upstream, creating larger pockets of nitrogen that carry the galinstan upwards in the riser tube.

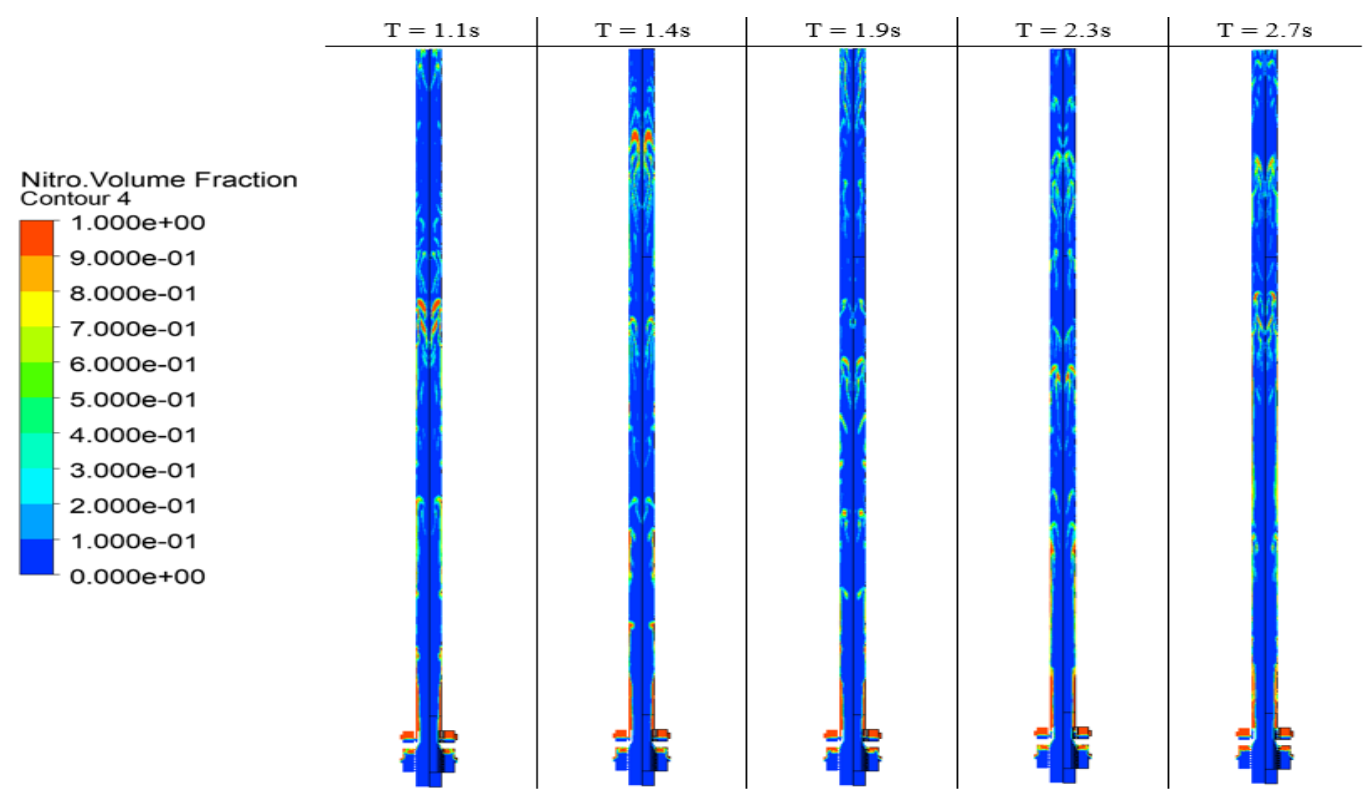

Fig. 4: Void Space Contours for the Gas-lift Injector and Riser. 


\section{Conclusion}

It is shown that the analytical model developed by Kassab et al. [8] for air-water can be altered to consider the properties properties of galinstan and nitrogen. The model was able to provide an initial prediction for the performance of a gas-lift lift pump in a vertical riser for a two-phase nitrogen and liquid metal system. The CFD results showed similar trends to those those predicted by the analytical model. The void function contours were presented to show the relationship between the the two-phase flow pattern occurring and the performance of the gas-lift pump. Thus, the results confirmed that the CFD approach is comparable to the $1 \mathrm{D}$ drift flux model. It should be noted that the present work is currently at the initial stage and both models need to be adjusted and validated with experimental data in order to be considered as suitable design tools. Further experimental study is required to refine these models so they can be used to predict the two-phase flow pumping performance required in the LMMHD power generation system.

\section{References}

[1] A. M. Quraishi, "Two-phase flow studies in liquid metal magnetohydrodynamic (MHD) power converison system of gravity type," Thesis, Aligarh Muslim University, 1999.

[2] M. Huleihil, "Power efficiency characteristics of MHD thermodynamic gas cycle," Therm. Sci. Eng. Prog., 2019.

[3] W. H. Ahmed, A. M. Aman, H. M. Badr, and A. M. Al-Qutub, "Air injection methods: The key to a better performance of airlift pumps,” Exp. Therm. Fluid Sci., vol. 70, pp. 354-365, 2016.

[4] H. Branover, A. Yakhot, and B. Sheva, "Testing of a low-temperature liquid-metal MHD power system," Energy Convers. Manag., vol. 22, pp. 163-169, 1982.

[5] D. G. Elliot, "Two-fluid magnetohydrodynamic cycle for nuclear-electric power conversion," ARS J., pp. 924-928, 1962.

[6] A. B. H. Branover, A. El-boher, E. Greenspan, "Promising applications of the liquid metal MHD energy conversion technology," in Proceedings of the 24th Intersociety, 1989, pp. 1051-1058.

[7] P. Satyamurthy, N. S. Dixit, T. K. Thiyagarajan, N. Venkatramani, A. M. Quraishi, and A. Mushtaq, "Two-fluid model studies for high density two-phase liquid metal vertical flows," Int. J. Multiph. Flow, vol. 24, no. 5, pp. 721737, 1998.

[8] S. Z. Kassab, H. A. Kandil, H. A. Warda, and W. H. Ahmed, "Air-lift pumps characteristics under two-phase flow conditions," Int. J. Heat Fluid Flow, vol. 30, no. 1, pp. 88-98, 2009.

[9] A. H. Stenning and C. B. Martin, "An analytical and experimental study of air-lift pump performance," J. Eng. Gas Turbines Power, vol. 90, no. 2, pp. 106-110, 1968.

[10] P. Griffith and G. B. Wallis, “Two-phase slug flow,” J. Heat Transfer, vol. 83, no. 3, pp. 307-318, 1961.

[11] S. E. Haaland, "Simple and explicit formulas for the friction factor in turbulent pipe flow," J. Fluids Eng. Trans. ASME, vol. 105, no. 1, pp. 89-90, 1983.

[12] E. Guerrero, F. Muñoz, and N. Ratkovich, "Comparison between eulerian and vof models for two-phase flow assessment in vertical pipes," CT\&F - Ciencia, Tecnol. y Futur., vol. 7, no. 1, pp. 73-84, 2017.

[13] ANSYS FLUENT 13 User's Guide, Ansys Fluent Theory Guide, no. November. 2013.

[14] T. Liu, P. Sen, C. C. J. Kim, and A. C. A. Measurements, "Characterization of nontoxic liquid-metal alloy galinstan for applications in microdevices," J. Microelectromechanical Syst., vol. 21, no. 2, pp. 443-450, 2012. 